\title{
Effect of normal saline flush injection into a bronchus on lung decellularization
}

\author{
Masahiro Yanagiya ${ }^{1}$, Kentaro Kitano ${ }^{1}$, Takuma Yotsumoto ${ }^{1}$, Daiya Takai ${ }^{2}$, Hiromichi Asahina ${ }^{1}$, \\ Kazuhiro Nagayama ${ }^{1}$, Jun Nakajima ${ }^{1}$
}

${ }^{1}$ Department of Thoracic Surgery, The University of Tokyo Graduate School of Medicine, Tokyo, Japan; ${ }^{2}$ Department of Clinical Laboratory, The University of Tokyo, Tokyo, Japan

Contributions: (I) Conception and design: M Yanagiya, K Kitano, J Nakajima; (II) Administrative support: K Kitano; (III) Provision of study materials or patients: K Kitano; (IV) Collection and assembly of data: M Yanagiya, T Yotsumoto, D Takai, H Asahina, K Nagayama; (V) Data analysis and interpretation: M Yanagiya, K Kitano, T Yotsumoto; (VI) Manuscript writing: All authors; (VII) Final approval of manuscript: All authors.

Correspondence to: Masahiro Yanagiya. Department of Thoracic Surgery, The University of Tokyo Graduate School of Medicine, 7-3-1 Hongo, Bunkyo-ku, Tokyo 113-8655, Japan. Email: YANAGIYAM-SUR@h.u-tokyo.ac.jp.

\begin{abstract}
Background: The aim of this study was to evaluate effect of normal saline flush injection into bronchus on creation of decellularized lung scaffolds.

Methods: Pigs were used: 3 lung grafts for decellularization with pre-treatment of normal saline injection into a bronchus, 3 for decellularization without pre-treatment and 3 treated as normal controls. We compared the characteristics of lung scaffolds created by each method.

Results: The pretreatment procedure significantly reduced the DNA content of lung grafts, suggesting effective removal of cellular components. However, this pretreatment also reduced the elastin contents of lung grafts.

Conclusions: Considering this characteristic of saline pretreatment, we must continue to look for better methods to produce ideal decellularized lung grafts.
\end{abstract}

Keywords: Regenerative medicine; lung tissue engineering; decellularization; extracellular matrix

Submitted Jul 01, 2019. Accepted for publication Nov 12, 2019.

doi: $10.21037 /$ jtd.2019.11.59

View this article at: http://dx.doi.org/10.21037/jtd.2019.11.59

\section{Introduction}

End-stage lung diseases continue to be fatal disorders $(1,2)$, for which there is no optimal treatment other than lung transplantation. Although lung transplantation would be definitively effective for patients with end-stage lung diseases, this treatment has many challenges such as a high number of complications, shortage of donors, and the requirement for administration of immunosuppressants for the remainder of patients' lives (3-5). To overcome these problematic issues, lung regeneration from recipientderived cells is needed (6). Recently, engineering of bioartificial lung scaffolds constructed by decellularization has been demonstrated as a promising procedure to realize lung regeneration, as they may potentially provide long- term graft survival without the use of immunosuppressive treatments $(7,8)$.

Various approaches have been developed to produce decellularized lung scaffolds, such as physical, enzymatic, and chemical approaches. Among these methods, chemical approaches have recently turned out to be the most cost effective. Triton X-100, sodium dodecyl sulfate (SDS), and 3-[(3-cholamidopropyl)dimethylammonio]-1propanesulfonate (CHAPS) are three candidate detergents (6). With regard to the effectiveness of removing cellular components, a combined method involving both SDS and Triton X-100 (hereafter referred to as the SDS/Triton X method) has been demonstrated as one of the most effective approaches $(9,10)$.

Although the SDS/Triton X method provides efficient 
decellularization, DNA content of decellularized grafts was only reduced to about $10-20 \%$ of normal lung grafts in large animals (10-12). To generate acellular lung grafts from large animals, cellular components must be eliminated as much as possible. Ideally, the DNA content of decellularized grafts should be approximately $0 \%$ of normal grafts. In this regard, one report suggested that instillation of water into the trachea before applying a detergent-based method might efficiently facilitate decellularization because it can remove trapped air from the lungs (12). Thus, this method should be effective in terms of reducing cellular components, as it will allow detergents to more easily spread into the peripheral parts of lungs without the presence of trapped air. However, no previous study investigated the effectiveness of instillation of water directly into a bronchus before a conventional detergent method. As such, we investigated the efficacy of water instillation into a bronchus before application a detergent method for decellularization of lung grafts.

\section{Methods}

\section{Study approval}

Porcine surgeries and subsequent experiments were approved by the Institutional Animal Care and Use Committee (P17-093) of the University of Tokyo. Institutional guidelines for the care and use of laboratory animals have been observed.

\section{Perfusion detergent decellularization of porcine lung grafts}

Pigs obtained by mating Yorkshire pigs with Landrace pigs (each weighing 15-25 kg) served as donors of lung grafts. Native lung grafts $(\mathrm{N}=9)$ were harvested after intravenous administration of $400 \mathrm{U} / \mathrm{kg}$ heparin. Among nine grafts, six left lungs were used for decellularization, while the other three right lungs were used as normal controls.

Lung decellularization was conducted using a previously reported SDS/Triton X method. Briefly, the main pulmonary artery was perfused with $0.5 \%$ SDS for $30 \mathrm{hr}$, deionized water for $12 \mathrm{hr}$, and $1 \%$ Triton X-100 for $12 \mathrm{hr}$ at a rate of $2 \mathrm{~mL} / \mathrm{min}$ (10). Finally, the main pulmonary artery was perfused with phosphate-buffered saline (PBS) for $48 \mathrm{hr}(10)$.

Normal saline flush was injected into a bronchus as shown in Figure 1. In the present study, normal saline (Otsuka, Tokyo, Japan) was used for injection. A total of
$2 \mathrm{~L}$ of normal saline was injected into a bronchus before performing detergent decellularization.

Three left grafts were pre-treated with $2 \mathrm{~L}$ of normal saline flush injected into a bronchus before performing the SDS/Triton $\mathrm{X}$ method (PreTx+; N=3). Another three left grafts were subjected to the SDS/Triton $\mathrm{X}$ method without saline pre-treatment (PreTx-; N=3). The remaining three right lung grafts did not undergo decellularization and were treated as normal grafts (Normal; $\mathrm{N}=3$ ).

In summary, grafts were divided into three different groups according to the three different treatments, as follows:

(I) Group PreTx+: SDS/Triton X method with pretreatment of bronchial saline injection.

(II) Group PreTx-: SDS/Triton X method without pretreatment of bronchial saline injection.

(III) Group Normal: no treatment.

\section{Histology}

Samples of tissues were fixed in paraformaldehyde, embedded in paraffin, and 5- $\mu \mathrm{m}$ sections were created. Hematoxylin and eosin staining was performed according to standard protocols.

For immunofluorescence staining, deparaffinized sections were initially subjected to antigen retrieval in citrate buffer (pH6, Sigma-Aldrich, St. Louis, MO) at $120^{\circ} \mathrm{C}$ for $20 \mathrm{~min}$. After antigen retrieval, sections were permeabilized with $0.1 \%$ Triton X-100 in PBS, washed with PBS, and blocked with $1 \%$ bovine serum albumin in PBS. Sections were incubated with primary antibodies overnight at $4^{\circ} \mathrm{C}$. The following day, sections were washed in PBS and incubated with an Alexa-conjugated secondary antibody for $30 \mathrm{~min}$. After staining, tissue slides underwent mounting with 4',6-diamigino-2-phenylidole (DAPI)-containing mounting media (Invitrogen, Carlsbad, CA). Primary and secondary antibodies are summarized in Table 1 .

\section{DNA extraction and qualification}

Tissue samples were digested in buffer containing $270 \mathrm{mM}$ $\mathrm{NaCl}, 1 \%$ SDS, $27 \mathrm{mM}$ Tris- $\mathrm{Cl}(\mathrm{pH} 8.0$ ), and $27 \mathrm{mM}$ ethylenediaminetetraacetic acid (EDTA, $\mathrm{pH}$ 8.0) with proteinase K $(10 \mu \mathrm{g} / \mathrm{mL}$, Sigma-Aldrich) and RNAse A $\left(10 \mu \mathrm{g} / \mathrm{mL}\right.$, Sigma-Aldrich) for $1 \mathrm{hr}$ at $45^{\circ} \mathrm{C}$ with gentle rotation. DNA was concentrated from the digest by phenolchloroform extraction and ethanol precipitation. Quantity and purity of DNA were assessed based on absorbances at 
Table 1 Antibody information

\begin{tabular}{|c|c|c|c|c|}
\hline Target & Host & Company & Catalog number & Dilution \\
\hline \multicolumn{5}{|c|}{ Primary antibodies } \\
\hline Laminin & Rabbit & Abcam & 11575 & $1: 100$ \\
\hline Fibronectin & Rabbit & Abcam & 2413 & $1: 100$ \\
\hline Collagen I & Rabbit & Abcam & 34710 & $1: 100$ \\
\hline \multicolumn{5}{|c|}{ Secondary antibody (Alexa Fluor ${ }^{\circledR} 546$-conjugated) } \\
\hline Rabbit IgG & Goat & Invitrogen & A11010 & $1: 500$ \\
\hline
\end{tabular}

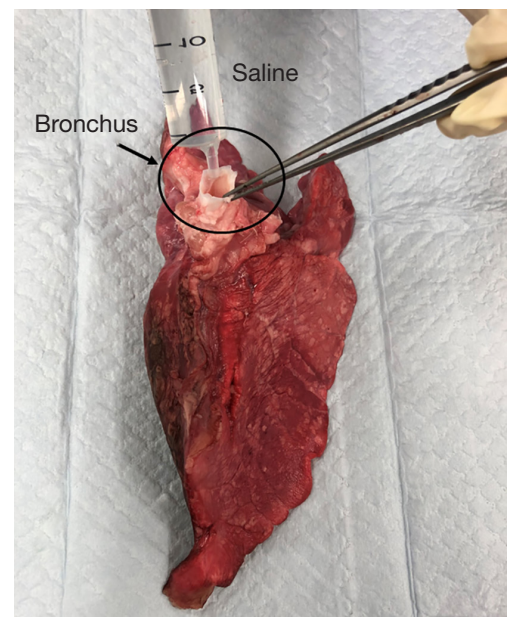

Figure 1 Pretreatment with normal saline injection into a bronchus. A total of $2 \mathrm{~L}$ of normal saline was instilled into the bronchus before decellularization.

260 and $280 \mathrm{~nm}$.

For quantification of sulfated glycosaminoglycan (sGAG) and elastin, samples were subjected to with Blyscan and Fastin assays (Biscolor, Carrickfergus, UK), which were performed according to the manufacturer's instructions. Sulfated glycosaminoglycan (sGAG) was extracted using a papain extraction reagent (Sigma-Aldrich) for $18 \mathrm{hr}$ at $65^{\circ} \mathrm{C}$.

\section{Statistics}

Values are expressed as mean \pm standard deviation. Statistical analysis was performed using Student's $t$ test. A P value $<0.05$ was defined as statistically significant for all tests.

\section{Results}

\section{Histology and immunofluorescence staining}

Hematoxylin and eosin staining revealed an obvious scarcity of cells in PreTx+ and PreTx- groups compared with the abundance of cells observed in the normal group (Figure 2). In addition, to confirm the preservation of extracellular matrix (ECM) proteins, we conducted immunofluorescence staining of laminin, fibronectin, and collagen I. Histological evaluation with immunofluorescence staining revealed no remaining nuclei in decellularized scaffolds (Figure 3). However, collagen I, laminin, and fibronectin were well preserved in decellularized lung scaffolds, regardless of pretreatment (Figure 3).

\section{Quantitative analyses of $D N A, s G A G$, and elastin contents}

Quantitative DNA analysis demonstrated a significant reduction of DNA content in both PreTx+ and PreTxgroups compared with the normal group $(35.1 \pm 17.1$ vs. $5,300 \pm 2,530 \mu \mathrm{g} / \mathrm{mg}$ wet tissue, $\mathrm{P}<0.001$ ) (Figure $4 A$ ). Moreover, DNA content was significantly lower in the PreTx+ group than in the PreTx- group (21.6 45.46 vs. $48.7 \pm 12.3 \mu \mathrm{g} / \mathrm{mg}$ wet tissue, $\mathrm{P}<0.05$ ) (Figure $4 B$ ).

Quantitative sGAG analysis revealed a significant reduction of sGAG in both PreTx+ and PreTx- groups compared with the normal group $(1.50 \pm 0.79$ vs. $5.69 \pm$ $2.13 \mu \mathrm{g} / \mathrm{mg}$ wet tissue, $\mathrm{P}<0.001$ ) (Figure $5 A$ ). However, there was no significant difference in sGAG content between PreTx+ and PreTx- groups (1.69 \pm 0.86 vs. $1.32 \pm$ $0.71 \mu \mathrm{g} / \mathrm{mg}$ wet tissue, $\mathrm{P}=0.337$ ) (Figure $5 B$ ).

Quantitative elastin analysis revealed a significant 


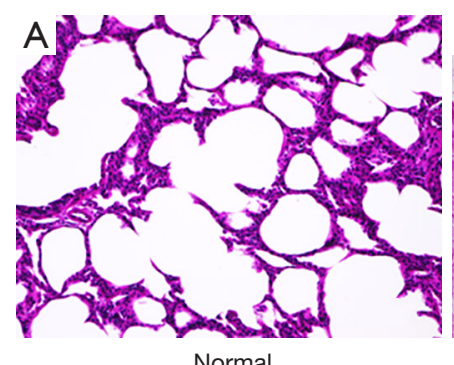

Normal

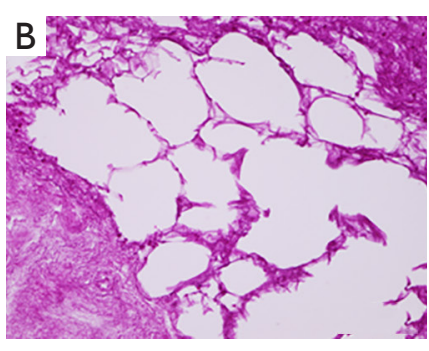

PreTx-

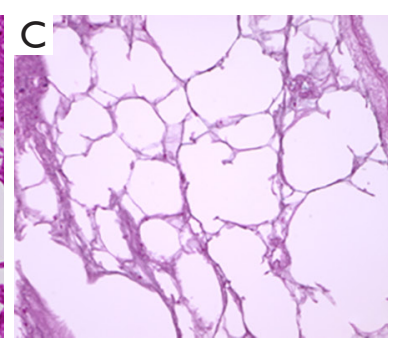

PreTx+

Figure 2 Hematoxylin and eosin staining of grafts from each group. Abundant cells were observed in the normal group (A), whereas only a few cells were observed in PreTx- and PreTx+ groups (B,C). Original magnification $\times 200$.
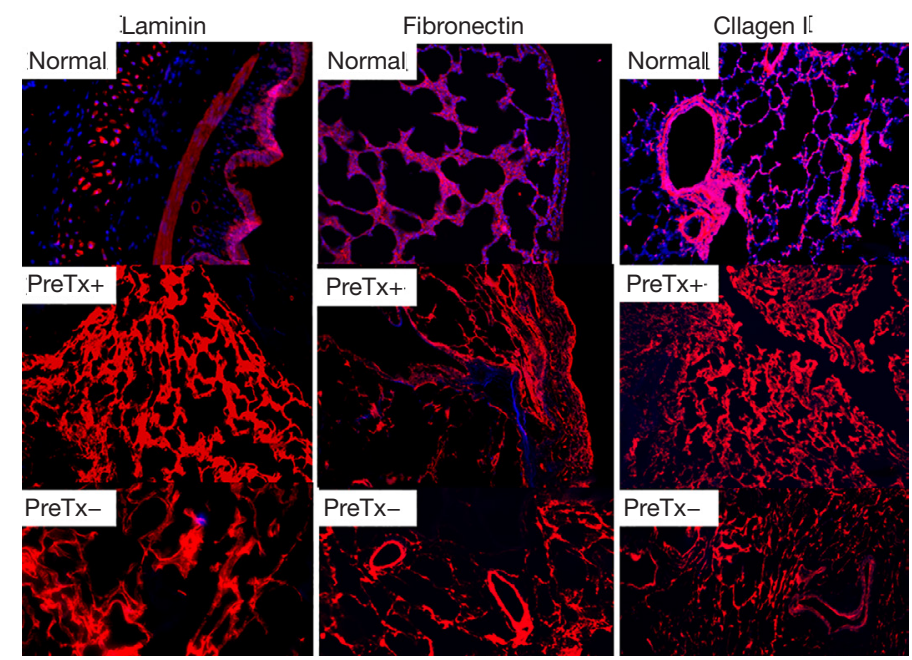

Figure 3 Immunofluorescence staining of grafts from each group. Tissues from the normal group exhibited abundant nuclei (blue) and ECM proteins (red), whereas tissues from both PreTx+ and PreTx-groups demonstrated an absence of nuclei, but preserved ECM proteins (red). Original magnification $\times 100$.

reduction of elastin in both PreTx+ and PreTx- groups compared with the normal group $(2.97 \pm 2.10$ vs. $14.3 \pm$ $3.24 \mu \mathrm{g} / \mathrm{mg}$ wet tissue, $\mathrm{P}<0.001$ ) (Figure 6A). Moreover, elastin content was significantly lower in the PreTx+ group than in the PreTx- group $(1.36 \pm 1.72$ vs. $4.59 \pm 0.50 \mu \mathrm{g} / \mathrm{mg}$ wet tissue, $\mathrm{P}<0.05$ ) (Figure $6 B$ ).

\section{Discussion}

Interestingly, the result of this study suggests that pretreatment with a saline flush injection into a bronchus could affect DNA and elastin contents of decellularized lung grafts. Although there have been many studies comparing decellularization methods, to the best of our knowledge, no previous research focused on pretreatment with a saline flush injection into a bronchus.

Quantification of DNA contents among PreTx+ and PreTx- groups, as shown in Figure 4, suggested that pretreatment lead to more efficient decellularization. This result was predicted based on the hypothesis that pretreatment with saline injection could remove trapped air in peripheral parts of lung grafts, thereby allowing detergents such as SDS and Triton X-100 to easily permeate grafts and achieve widespread distribution. Peripheral permeation of detergents should radically improve the removal of cells and, indeed, our results support this hypothesis. Thus, in terms of cell removal, this pretreatment method would be useful for decellularization of lung grafts.

However, our results also revealed that saline 

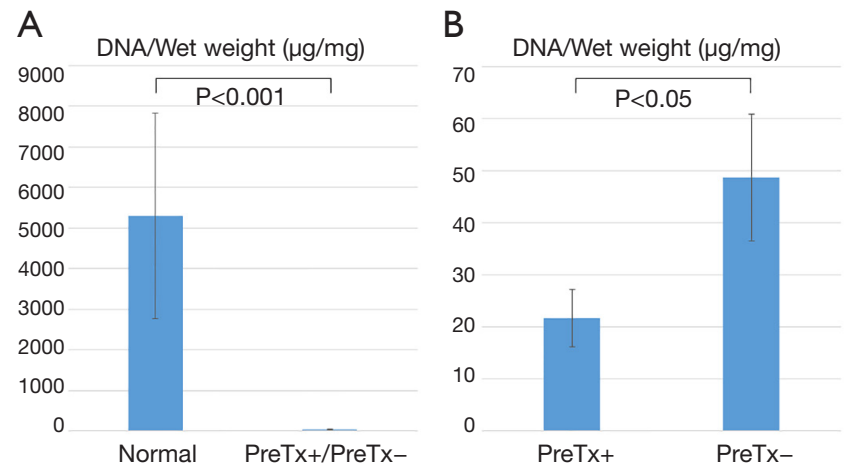

Figure 4 Quantitative comparison of quantitative DNA contents among groups. (A) DNA content was significantly lower in PreTx+ and PreTx groups compared with the normal group $(35.1 \pm 17.1$ vs. 5,300 $\pm 2,530 \mu \mathrm{g} / \mathrm{mg}$ wet tissue, $\mathrm{P}<0.001$ ). (B) DNA content was significantly lower in the Pre $\mathrm{T} x+$ group than in the PreTx-group (21.6 \pm 5.46 vs. $48.7 \pm 12.3 \mu \mathrm{g} / \mathrm{mg}$ wet tissue, $\mathrm{P}<0.05)$.

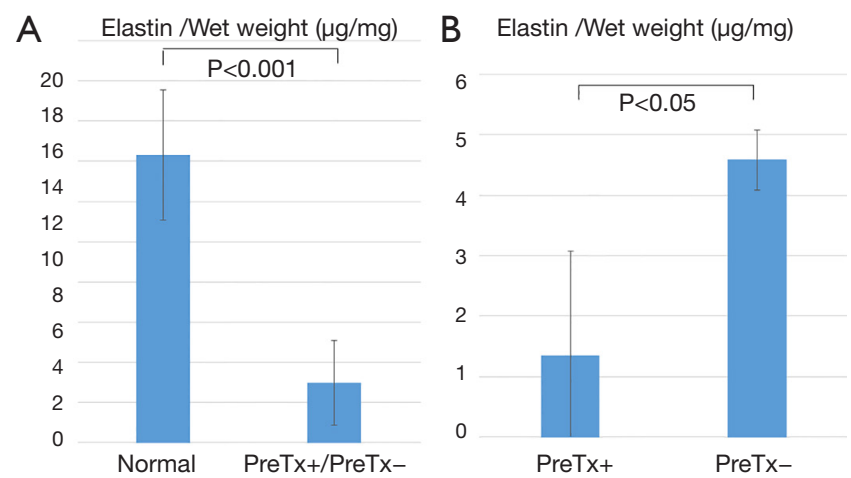

Figure 6 Quantitative comparison of elastin contents among groups. (A) Elastin contents were significantly lower in PreTx+ and PreTx-groups compared with the normal group $(2.97 \pm 2.10$ vs. $14.3 \pm 3.24 \mu \mathrm{g} / \mathrm{mg}$ wet tissue, $\mathrm{P}<0.001$ ). (B) Elastin content was significantly lower in the PreTx+ group than in the PreTx- group (1.36 \pm 1.72 vs. $4.59 \pm 0.50 \mu \mathrm{g} / \mathrm{mg}$ wet tissue, $\mathrm{P}<0.05)$.

pretreatment could lead to a significant reduction of elastin, as shown in Figure 6B. This is unfavorable, as elastin is a crucial extracellular matrix (ECM) protein that provide lungs with elasticity, resilience, and deformability (13). Degradation of elastin fibers is considered one of the major factors for development of emphysema (14), as elastin is essentially required for breathing. Thus, decellularized lung scaffolds with preserved elastin are ideal. From this point of view, pretreatment with saline injection into the bronchus would not be favorable.
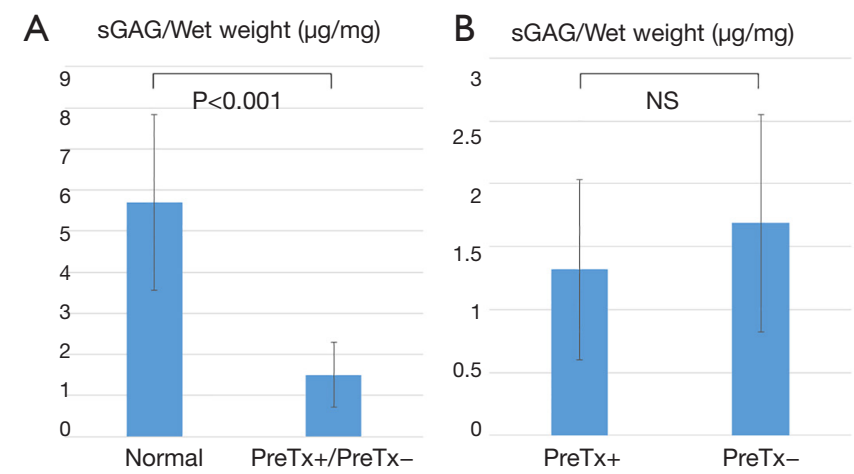

Figure 5 Quantitative comparison of sGAG contents among groups. (A) Sulfated glycosaminoglycans (sGAG) content was significantly lower in PreTx+ and PreTx-

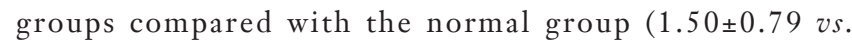
$5.69 \pm 2.13 \mu \mathrm{g} / \mathrm{mg}$ wet tissue, $\mathrm{P}<0.001)$. (B) There was no significant difference in sGAG content between PreTx+ and PreTx-groups $(1.69 \pm 0.86$ vs. $1.32 \pm 0.71 \mu \mathrm{g} / \mathrm{mg}$ wet tissue, $\mathrm{P}=0.337$ ).

The mechanism of saline pretreatment on reduction of elastin still remained unknown. One recent interesting report may provide us a clue to understand this phenomenon (15). The report suggested that lung edema could lead to disorganization of ECM proteins (15). In our pretreatment method, we injected $2 \mathrm{~L}$ of normal saline into a bronchus of a graft just after the harvest. Thus, we might have created the graft edema by means of normal saline injection, which finally caused reduction of elastin.

With regard to the reduction of elastin content, the SDS/Triton X method also significantly decreased elastin contents of grafts, as shown in Figure 6A. This result was consistent with previous reports (10). Efficacy of the SDS/ Triton X method has not only been characterized by reduced DNA content, but also reduced elastin and sGAG $(10,16)$. Although we acknowledge that the SDS/Triton $\mathrm{X}$ method could preserve some ECM proteins as shown in Figure 3, this method may not generate acellular lung scaffolds with well-preserved ECM proteins, as it led to a significant reduction of elastin. Thus, we must continue to search for methods to create decellularized lung scaffolds that preserve as much elastin as possible.

CHAPS may be a promising alternative for preparing decellularized lung scaffolds with well-preserved ECM proteins, as previous reports suggest that CHAPS-based decellularization is less likely to reduce ECM proteins such as elastin $(8,17)$. However, CHAPS-based decellularization is reportedly less effective for the removal of cells compared 
with the SDS/Triton X method (8).

To overcome this disadvantage of CHAPS-based decellularization, pretreatment with saline injection into a bronchus could be of great value. Indeed, pretreatment with saline injection should complement the CHAPSbased method to facilitate more efficient cell removal, thus potentially producing more optimal decellularized lung scaffolds. Moreover, such a pretreatment could serve as a strong aid to reduce cell components in combination with other promising methods to preserve ECM proteins.

\section{Conclusions}

We evaluated the efficacy of pretreatment with saline injection into a bronchus on lung decellularization. This pretreatment was effective in terms of reducing cellular components. However, this procedure may also reduce elastin, an important ECM protein. Considering the pros and cons of this pretreatment, future research should be conducted to optimize this procedure for production of an ideal lung decellularized scaffold.

\section{Acknowledgments}

The authors thank the staff of the Bioscience Research Building at the University of Tokyo for assistance with animal surgeries, as well as Edanz Group (www. edanzediting.com/ac) for editing a draft of this manuscript.

Funding: This work was supported by JSPS KAKENHI Grant Number JP17H06274.

\section{Footnote}

Conflicts of Interest: The authors have no conflicts of interest to declare.

Etbical Statement: The authors are accountable for all aspects of the work in ensuring that questions related to the accuracy or integrity of any part of the work are appropriately investigated and resolved. The study was approved by the Institutional Animal Care and Use Committee (P17-093) of the University of Tokyo.

\section{References}

1. Murphy SL, Xu J, Kochanek KD, Arias E. Mortality in the United States, 2017. NCHS Data Brief.
2018;328. Hyattsville, MD: National Center for Health Statistics. 2018.

2. Raghu G, Collard HR, Egan JJ, et al. An official ATS/ ERS/JRS/ALAT statement: idiopathic pulmonary fibrosis: evidence-based guidelines for diagnosis and management. Am J Respir Crit Care Med 2011;183:788-824.

3. Munshi L, Keshavjee S, Cypel M. Donor management and lung preservation for lung transplantation. Lancet Respir Med 2013;1:318-28.

4. Nathan SD. The future of lung transplantation. Chest 2015;147:309-16.

5. Sato M, Waddell TK, Wagnetz U, et al. Restrictive allograft syndrome (RAS): a novel form of chronic lung allograft dysfunction. J Heart Lung Transplant 2011;30:735-42.

6. Moser PT, Ott HC. Recellularization of organs: what is the future for solid organ transplantation?. Curr Opin Organ Transplant 2014;19:603-9.

7. Tapias LF, Ott HC. Decellularized scaffolds as a platform for bioengineered organs. Curr Opin Organ Transplant 2014;19:145-52.

8. Petersen TH, Calle EA, Colehour MB, Niklason LE. Matrix composition and mechanics of decellularized lung scaffolds. Cells Tissues Organs 2012;195:222-31.

9. Ott HC, Clippinger B, Conrad C, et al. Regeneration and orthotopic transplantation of a bioartificial lung. Nat Med 2010;16:927-33.

10. Zhou H, Kitano K, Ren X, et al. Bioengineering Human Lung Grafts on Porcine Matrix. Ann Surg 2018;267:590-8.

11. Bonvillain RW, Scarritt ME, Pashos NC, et al. Nonhuman primate lung decellularization and recellularization using a specialized large-organ bioreactor. J Vis Exp 2013;(82):e50825.

12. Weymann A, Patil NP, Sabashnikov A, et al. Perfusion-Decellularization of Porcine Lung and Trachea for Respiratory Bioengineering. Artif Organs 2015;39:1024-32.

13. Mithieux SM, Weiss AS. Elastin. Adv Protein Chem 2005;70:437-61.

14. Stockley RA. Neutrophils and protease/ antiprotease imbalance. Am J Respir Crit Care Med 1999;160:S49-52.

15. Pelosi P, Rocco PR, Negrini D, et al. The extracellular matrix of the lung and its role in edema formation. An Acad Bras Cienc 2007;79:285-97. 
16. Kitano K, Schwartz DM, Zhou H, et al. Bioengineering of functional human induced pluripotent stem cell-derived intestinal grafts. Nat Commun 2017;8:765.

17. Wallis JM, Borg ZD, Daly AB, et al. Comparative

Cite this article as: Yanagiya M, Kitano K, Yotsumoto T, Takai D, Asahina H, Nagayama K, Nakajima J. Effect of normal saline flush injection into a bronchus on lung decellularization. J Thorac Dis 2019;11(12):5321-5327. doi: 10.21037/jtd.2019.11.59 assessment of detergent-based protocols for mouse lung de-cellularization and re-cellularization. Tissue Eng Part C Methods 2012;18:420-32. 